\title{
Simulation Analyze the Dice and Shape of the Dicer Based on ADAMS
}

\author{
Yingsa Huang, Jianping Hu, Deyong Yang, Xiuping Shao, and Fa Liu \\ Key Laboratory of Modern Agricultural Equipment and Technology, Ministry of \\ Education\&Jiangsu Province, Jiangsu University, Zhenjiang 212013, China \\ huangyingsa_148@126.com
}

\begin{abstract}
The Fruits and Vegetables diversacut dicer is composed of impeller roller, circular knivess and crosscut knives. The mismatch of the rotationl speed ratio and the unsuitable central position arrangement between the impeller and the crosscut will directly affect the quality of the diced section which is apt to appear marked arc, incline and so forth. Therefore, In this paper, using the virtual prototype technology to build the 3D model of fruits and vegetables diversacut dicer, and import it into ADAMS (dynamic analysis software of mechanical system) to simulation analyze the different shape of diced section when changing the rotationl speed ratio from 0.11 to 0.22 and the central horizontal spacing between the impeller and the crosscut vary from $260 \mathrm{~mm}$ to $310 \mathrm{~mm}$. The results indicated that the mismatch between the impeller speed and the crosscut speed is the main factor which causes the incline surface during dicing process. If the central horizontal spacing between the impeller and the crosscut set at $280 \mathrm{~mm}, 285 \mathrm{~mm}, 290 \mathrm{~mm}, 295 \mathrm{~mm}, 300 \mathrm{~mm}$, and the rotationl speed ratio set at $0.11,0.13,0.16,0.18(0.19), 0.21$, the optimal diced section can be acquired so as to provide a basis for improving the diced quality.
\end{abstract}

Keywords: Dice, Diced section, Shape, ADAMS, Simulation.

\section{Introduction}

In recent years, to satisfy the requirement for large number of fresh-cut fruits and vegetables in the food processing industry in our country, a new Fruits and Vegetables diversacut dicer was designed absorbed the traditional handiwork technolgies and the advanced technology from the foreign countries. This dicer can solve the problem of unable for automatical feed, automatic cut and smooth discharge which existed in the traditional dicer very well. Additionally, it enhanced work efficiency, reduced working strength and satisfied the scale-production request in fruit and vegetable processing industry[1,2]. But there still existing many shortage, for example the mismatch of the rotationl speed ratio and the unsuitable central position arrangement between the impeller and the crosscut directly affect the quality of the diced section which is apt to appear marked arc, incline and so forth. This paper applied virtual prototyping technology, carried out kinematic simulation and analysis on the dicer to 
Analyze the main factors which influenced the quality of the diced section, so as to provide evidence for improving the quality of diced section.

\section{Structures and Work Principles of the Diversacut Dicer}

The diversacut dicer is composed of impeller roller, circular knives and crosscut knives, which is shown in Figure 1. The dicer adopted diversacut technology, including feeding by rotary propeller, slicing by centrifugal cutting method, cutting strips by circular knives and finally cut by crosscut knives. The main parameters of the dicer was set that the inner diameter of impeller roller is $\mathrm{R} 1=200 \mathrm{~mm}$ and 6 paddles in the impeller the radius of the circular knives is $\mathrm{R} 2=45 \mathrm{~mm}$ and the radius of the track of the crosscut knifepoint is R3 $110 \mathrm{~mm}$. The amount of the circular and the crosscut knives can be adjusted according to the cutting specifications.

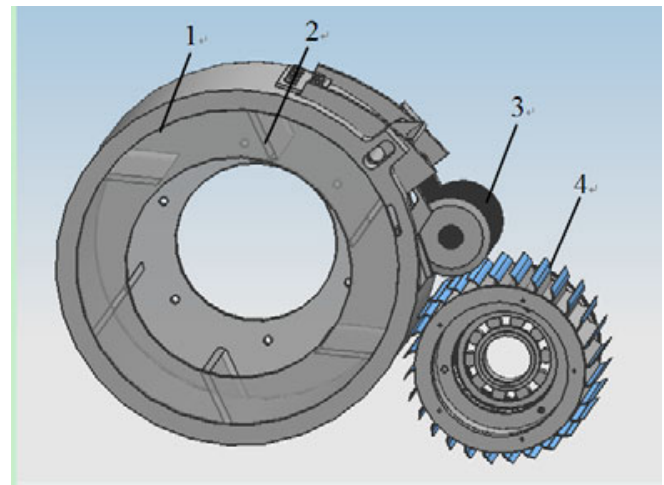

1. Impeller roller 2. Paddle 3. Circular knives 4. Crosscut knives

Fig. 1. The 3D model of the dicer machinery

\section{Virtual Prototype Modeling}

According to the designed parameters of the diversacut dicer, the 3D solid model of the machine part was established in UG4.0 and the assembly was completed, thereby established the virtual prototyping parameterized model[3,4]. Then imported the model into ADAMS using of the Model Data exchange interface which provided by UG4.0 and the dynamic analysis software of mechanical system ADAMS. After the successfully transformation of the model, the constraints was added in ADAMS including Fixed pair joint between the slicer and the impeller roller, Fixed pair joint between the slicer and the crosscut knives holder, Fixed pair joint between the crosscut knives and the crosscut knives spindle, Revolute pair joint between the crosscut knives holder and the crosscut knives spindle, Translation pair joint between the slicer and the ground. Then added motions on the model including a rotational motion and a Translational motion[3-5]. Then moved and rotated the model in order that the bottom of the slicer is at the origin and the slicer is along the $\mathrm{Y}$-axis. 


\section{Simulation Analyzing the Shape of Diced Section}

\subsection{Simulating Condition Setting}

The rotational velocity of the impeller roller was set at $120 \mathrm{r} / \mathrm{min}$ while the rotationl speed ratio between the impeller roller and the crosscut knives which is indicated by was set from 0.11 to 0.22 and the specification of the diced product was $20 \mathrm{~mm} \times 20 \mathrm{~mm} \times 20 \mathrm{~mm}$.

In order that the deformation of the fruits and vegetables material during the cutting process is lesser and the simulating result conforms with the actual result, the structural arrangement of the machine should be compact, what is to say is that the triangular region between the impeller roller, the circular knives and crosscut knives is small, as shown in figure 2. The arrangement of the impeller roller and the crosscut knives is shown in Table 1.

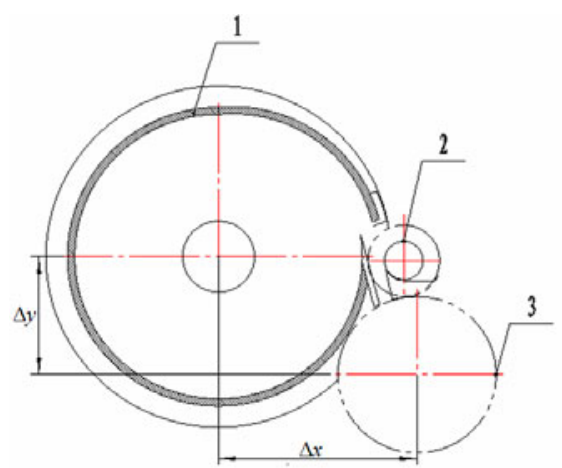

Fig. 2. The structural arrangement of the machine

Table 1. The arrangement of the impeller roller and the crosscut knives

\begin{tabular}{cc}
\hline horizontal spacing $\Delta x /(\mathrm{mm})$ & vertically spacing $\Delta y /(\mathrm{mm})$ \\
\hline 260.00 & 188.75 \\
265.00 & 183.19 \\
270.00 & 174.03 \\
275.00 & 167.67 \\
280.00 & 162.26 \\
285.00 & 161.36 \\
290.00 & 161.98 \\
295.00 & 159.86 \\
300.00 & 159.86 \\
305.00 & 159.86 \\
310.00 & 154.68 \\
\hline
\end{tabular}




\subsection{Analysis of the Simulation Results}

The cutting experiment indicated that the irregularity of the diced section was caused during the process of dicing by the crosscut knives. Especially, the rotationl speed ratio and the central position arrangement between the impeller roller and the crosscut knives greatly affect the quality of the diced section. In the simulation analysis, the reverse method that the crosscut knives moves upward along the slice blade while rotating was adopted. The coordinate curve of the crosscut knifepoint can be got, which is the shape curve of the diced section, then provided the basis for optimizing the arrangement and determine the transmission of the dicer[4-7].

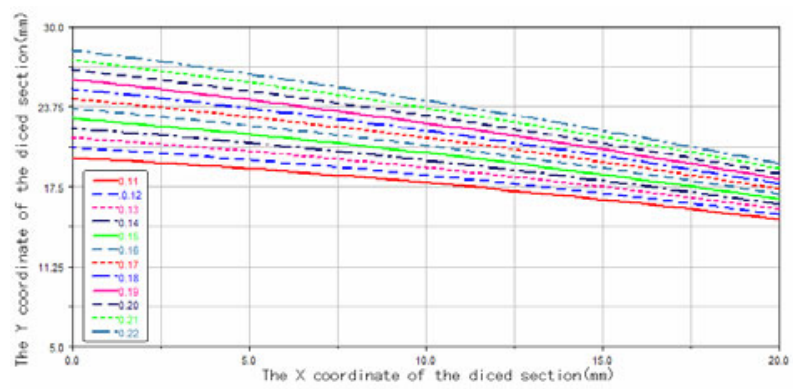

Fig. 3. The shape curve of the diced section when $\Delta x=260 \mathrm{~mm}$

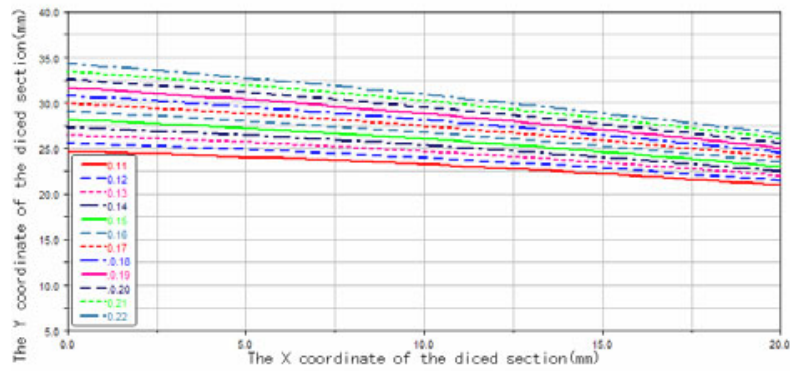

Fig. 4. The shape curve of the diced section when $\Delta x=265 \mathrm{~mm}$

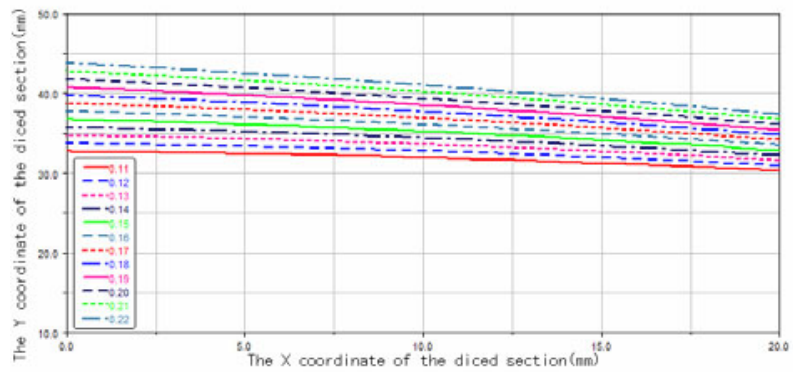

Fig. 5. The shape curve of the diced section when $\Delta x=270 \mathrm{~mm}$ 


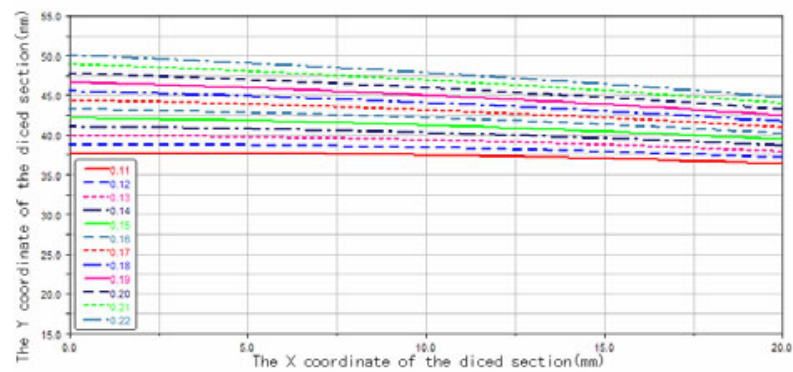

Fig. 6. The shape curve of the diced section when $\Delta x=275 \mathrm{~mm}$

When the arrangement of the impeller roller and the crosscut knives was set that the horizontal spacing $\Delta x=260 \mathrm{~mm} \sim 310 \mathrm{~mm}$, the rotationl speed ratio between the impeller roller and the crosscut knives was set that ${ }^{n_{1} / n_{3}}=0.11 \sim 0.22$, Figure 3 to Figure 13 respectively shown the simulation result of the shape curve of the diced section.

As can be seen from the Figure 3,4,5,6, when the horizontal spacing between the impeller roller and the crosscut knives is $260 \mathrm{~mm} \sim 275 \mathrm{~mm}$, choosing the rotationl speed ratio from 0.11 to 0.22 , the diced section appears incline surface,and the larger of the ratio,the more obviously of the incline.

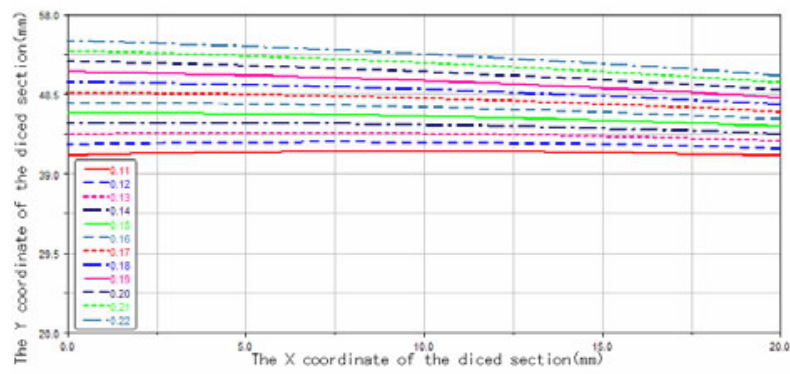

Fig. 7. The shape curve of the diced section when $\Delta x=280 \mathrm{~mm}$

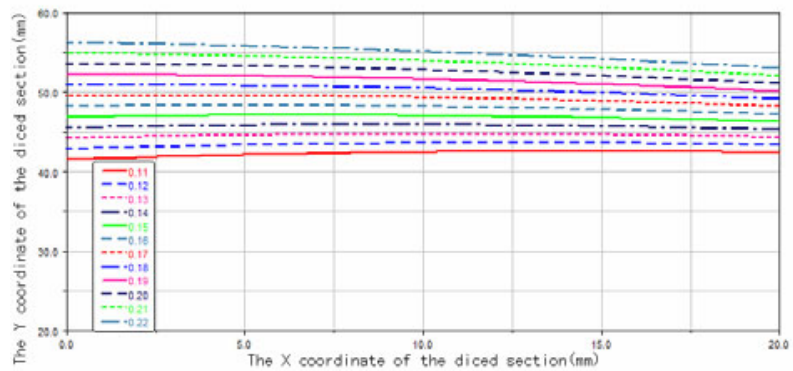

Fig. 8. The shape curve of the diced section when $\Delta x=285 \mathrm{~mm}$ 


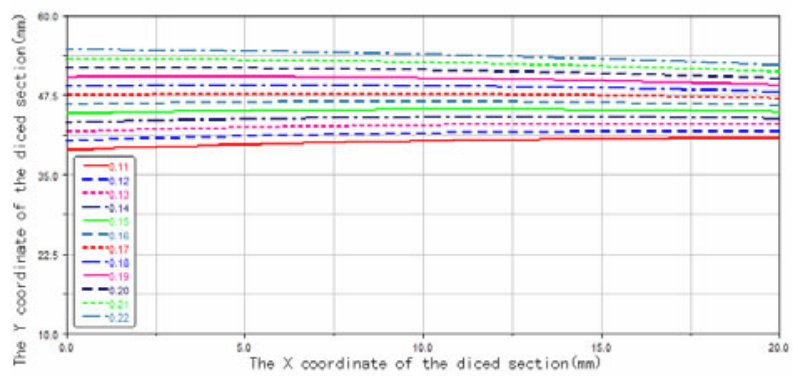

Fig. 9. The shape curve of the diced section when $\Delta x=290 \mathrm{~mm}$

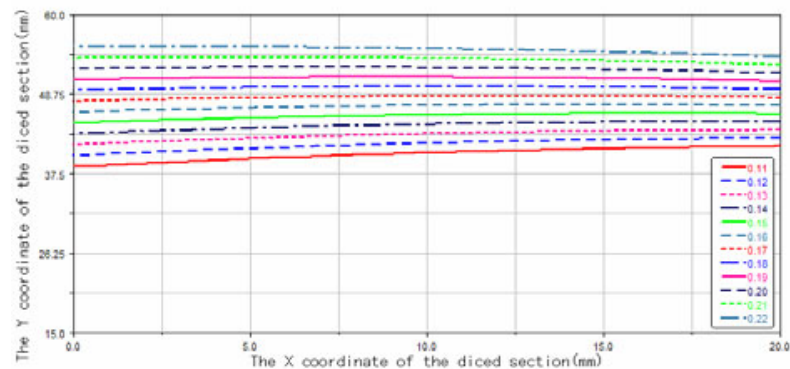

Fig. 10. The shape curve of the diced section when $\Delta x=295 \mathrm{~mm}$

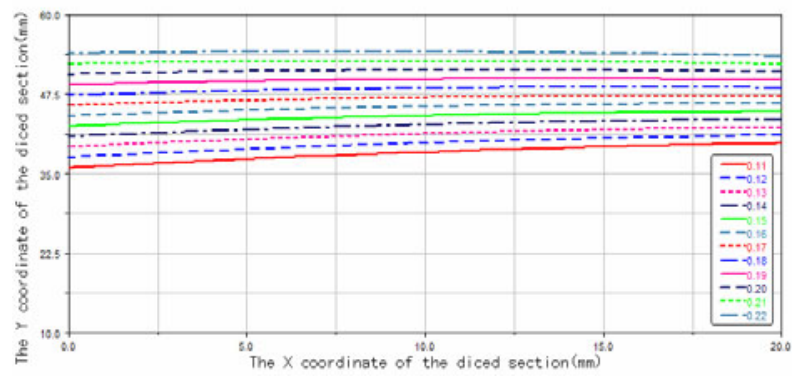

Fig. 11. The shape curve of the diced section when $\Delta x=300 \mathrm{~mm}$

As can be seen from the Figure 7 to Figure 11, when the horizontal spacing between the impeller roller and the crosscut knives is $280 \mathrm{~mm} \sim 300 \mathrm{~mm}$, choosing the rotationl speed ratio from 0.11 to 0.22 , the ideal diced section can be acquired, and it appears marked arc surface.

As can be seen from the Figure 12,13, when the horizontal spacing between the impeller roller and the crosscut knives is $305 \mathrm{~mm} \sim 310 \mathrm{~mm}$, choosing the rotationl speed ratio from 0.11 to 0.22 , the diced section appears incline surface,and the larger of the ratio,the less obviously of the incline. 


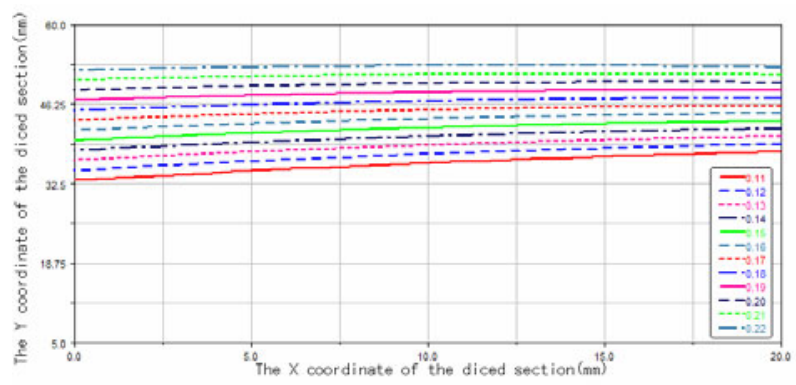

Fig. 12. The shape curve of the diced section when $\Delta x=305 \mathrm{~mm}$

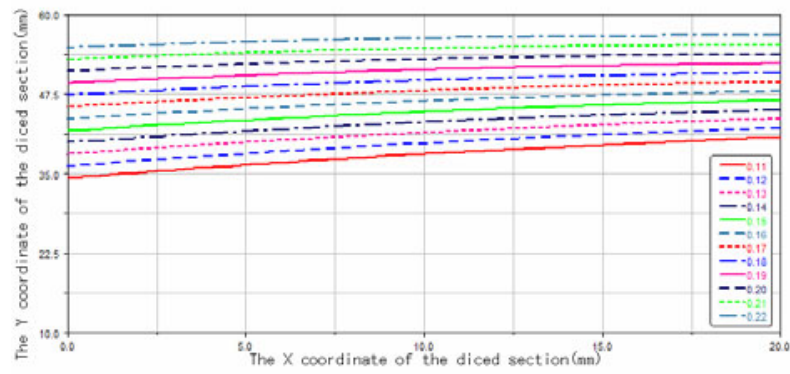

Fig. 13. The shape curve of the diced section when $\Delta x=310 \mathrm{~mm}$

\subsection{Irregularity Degree Analyzation of the Diced Section}

In this paper, the quality of the diced section was measured by the Irregularity degree that the vertical distance between the section peak and the bottom ' $h$ ', as shown in Figure 14.

Through analyzing the data of the simulation results, the Irregularity degree of the diced section under different rotationl speed ratio and central position arrangement between the impeller and the crosscut was got, which is shown in Figure 15.
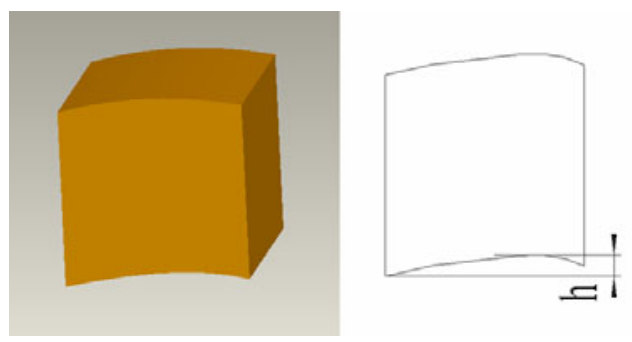

Fig. 14. The Irregularity degree of the diced section 


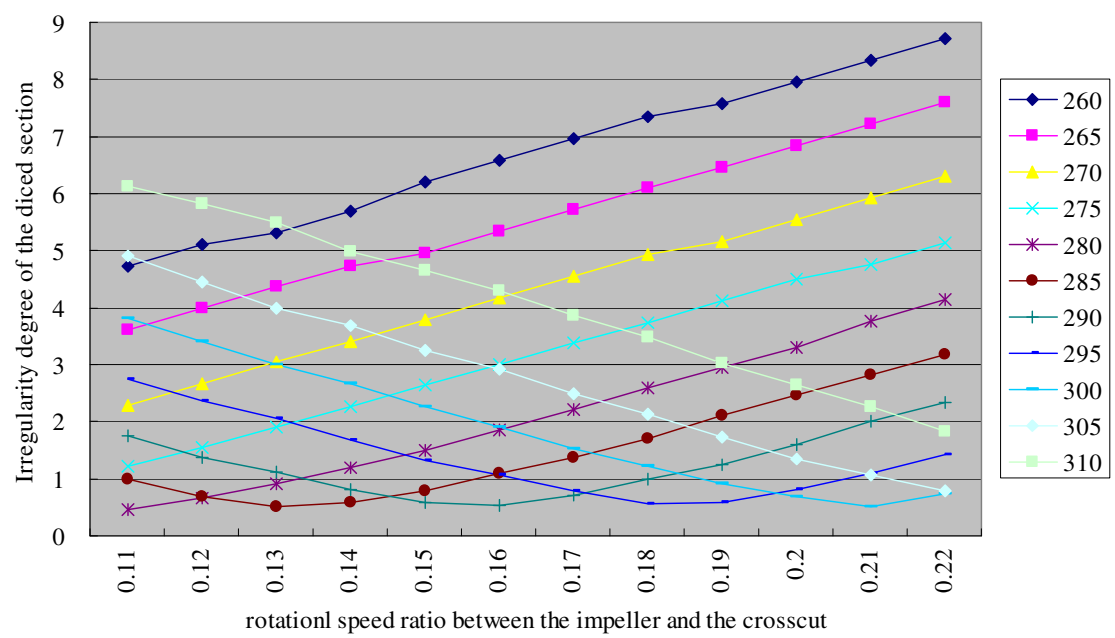

Fig. 15. the Irregularity degree of the diced section under different rotationl speed ratio and central position arrangement between the impeller and the crosscut

As can be seen from the Figure 15, if the central horizontal spacing between the impeller and the crosscut set at $280 \mathrm{~mm}, 285 \mathrm{~mm}, 290 \mathrm{~mm}, 295 \mathrm{~mm}, 300 \mathrm{~mm}$, when the rotationl speed ratio set at $0.11,0.13,0.16,0.18(0.19), 0.21$, the irregularity degree is the least, and the quality of the diced section is the best.

\section{Conclusion}

1. Through kinematics simulation of the cutting mechanism, come to a conclusion that when the horizontal spacing between the impeller roller and the crosscut knives is $260 \mathrm{~mm} \sim 275 \mathrm{~mm}$ and $305 \mathrm{~mm} \sim 310 \mathrm{~mm}$, the rotationl speed ratio adjust from 0.11 to 0.22 , the ideal diced section can't be acquired. What's more, the diced section appears obviously incline surface.

2. When the horizontal spacing between the impeller roller and the crosscut knives is $280 \mathrm{~mm} \sim 300 \mathrm{~mm}$, the rotationl speed ratio adjust from 0.11 to 0.22 , the ideal diced section can be acquired, the diced section appears marked arc surface. The simulating result provided a basis for the central position arrangement between the impeller roller and the crosscut knives.

3. Through the Irregularity degree analyzation of the diced section, the ideal parameter combination that if the central horizontal spacing between the impeller and the crosscut set at $280 \mathrm{~mm}, 285 \mathrm{~mm}, 290 \mathrm{~mm}, 295 \mathrm{~mm}, 300 \mathrm{~mm}$, the rotationl speed ratio should be set at $0.11,0.13,0.16,0.18(0.19), 0.21$ was acquired. In this parameters, the quality of the diced section is best, as provided a basis for optimized the working parameter of the dicer next. 


\section{Acknowledgements}

This work was financially supported by Tackling key Problems of Science and Technology of Jiangsu Province (BE2008385).

\section{References}

1. Bi, W.: Studies on Slicing Techniques and Material Characteristic of Lotus Root. D. Jiangsu University (2006)

2. Wei, E.: The Simulation and Optimization on the New Slicing Machine of Lotus Root Based on Virtual Prototype Technology. Jiangsu University (2008)

3. Kong, Z.: Design of Cotton Transplanter Based on Virtual Prototype. Shandong University of Technology (2006)

4. Li, W., Li, J., Zhang, J., et al.: Optimization Design and Simulations of the apple-pickingrobot arm. Journal of Beijing University of Technology 35(6), 721-726 (2009)

5. Wang, C., Tan, L.: Study on a Virtual Prototype Based Hay Highly Compressing Process. Transactions of the Chinese Society for Agricutural Machinery 36(3), 99-101 (2005)

6. Wang, W., Dou, W., Wang, C., et al.: Parameter Analysis of the Planting Process of 2ZT-2 Beet Transplanter. Transactions of the Chinese Society for Agricutural Machinery 40(1), 69-73 (2009)

7. Zhang, Y., Ou, Y., Mou, X.: Virtual Test on the Finger-Chain type Sugarcane-Lifter Based on ADAMS 25(7), 88-93 (2009) 